\title{
Concordancia entre nivel de inserción clínico y examen radiográfico para diagnóstico de periodontitis crónica
}

\author{
Concordance between Clinical Attachment Level and Radiographic \\ Examination for Diagnosis of Chronic Periodontitis
}

\author{
DÍAZ CABALLERO AJ* \\ GONZÁLEZ MARTÍNEZ FD** \\ ARÉVALO TOVAR LL***
}

\begin{abstract}
Díaz Caballero AJ, González Martínez FD, Arévalo Tovar LL. Concordancia entre nivel de inserción clínico y examen radiográfico para diagnóstico de periodontitis crónica. Av Periodon Implantol. 2012; 24, 2: 95-102.
\end{abstract}

\begin{abstract}
RESUIMEN
Objetivo. Determinar la concordancia y relación entre el nivel de inserción clínico periodontal y el examen radiográfico en pacientes con periodontitis crónica.

Métodos. Estudio de corte transversal en 135 participantes mayores de 16 años, seleccionados de forma aleatoria, con diagnóstico de periodontitis crónica. De los cuales se obtuvieron 352 radiografías periapicales en 1292 sitios interproximales. Se midieron las alturas de las crestas alveolares con una regla milimetrada. Además se examinaron el tipo de defecto, la continuidad de la lámina dura y el ensanchamiento del espacio del ligamento periodontal. Los datos fueron analizados a través de medidas de tendencia central y dispersión. Para cuantificar la concordancia se utilizó la prueba Kappa y para las relaciones se utilizaron las razones de odds con intervalos de confianza del $95 \%$, además para establecer significancia se utilizó la prueba $\chi^{2}$ asumiendo un límite de decisión de 0,05.

Resultados. Se encontró una relación estadísticamente significativa entre los niveles de inserción 4, 5 y $6 \mathrm{~mm}$ y la pérdida ósea radiográfica. Para las demás relaciones no hubo significancia estadística. Con respecto al tipo de defecto, el horizontal fue el más común con un $23 \%$ de los sitios examinados. Conclusión. Para el diagnóstico de periodontitis crónica no es suficiente con la valoración del examen radiográfico, porque en niveles de inserción 1,2 y $3 \mathrm{~mm}$ no se presenta pérdida ósea visible. Es evidente que al utilizar solamente este método diagnóstico se aumentaría los falsos negativos, subregistrándose la enfermedad.
\end{abstract}

PALABRAS CLAVE: Periodontitis crónica, pérdida de la inserción periodontal, resorción ósea, radiografía dental (Decs Bireme).

\section{SUMIMARY}

Objective. Determine the concordance and relationship between periodontal clinical attachment level and radiographic examination in patients with chronic periodontitis.

Methods. Cross-sectional study of 135 participants over 16 years, randomly selected, diagnosed with chronic periodontitis. Of which 352 were obtained in 1292 periapical interproximal sites. It was measured the height of the ridge with a millimeter ruler. Furthermore, were examined the type of

$*$

Odontólogo. Universidad de Cartagena. Especialista en Periodoncia. Universidad Javeriana. Magister en Educación Universidad del Norte. Estudiante doctorado en Ciencias Biomédicas Universidad de Cartagena. Profesor titular, Universidad de Cartagena. Director Grupo de investigaciones GITOUC.

$* *$ Odontólogo. Universidad de Cartagena. Especialista en investigación social. Universidad de Cartagena. Magíster en Salud Pública. Universidad Nacional de Colombia. Jefe del Departamento de Investigación. Facultad de Odontología. Universidad de Cartagena.

*** Odontóloga. Universidad de Cartagena. Especialista en Periodoncia. Universidad de Buenos Aires, Argentina. Profesora titular Universidad de Cartagena. Decana Facultad de Odontología, Universidad de Cartagena. 
defect, the continuity of the lamina dura and widening of the periodontal ligament space. The data were analyzed using measures of central tendency and dispersion. To quantify the correlation test was used for Kappa and relationships were used odds ratios with confidence intervals of $95 \%$, and was used to establish significance Chi-square test assuming a decision limit of 0.05.

Results. There was a statistically significant relationship between attachment levels 4,5 and $6 \mathrm{~mm}$ and radiographic bone loss. For the other relationships were not statistically significant. Regarding the type of defect, the horizontal pattern loss was the most common with $23 \%$ of the sites examined. Conclusion. For the diagnosis of chronic periodontitis is not enough with the assessment of radiographic examination, because attachment levels 1,2 and $3 \mathrm{~mm}$ bone loss occurs not visible. It is clear that using only this method of diagnosis would increase the false negative underreported disease.

KEY WORDS: Chronic periodontitis, periodontal attachment loss, bone resorption, dental radiography (Mesh Database).

Fecha de recepción: Noviembre de 2010.

Fecha de aceptación: 27 de enero de 2011.

\section{INTRODUCCIÓN}

La periodontitis representa la expresión clínica de una serie de entidades que tienen en común la destrucción de los tejidos de soporte del diente, incluyendo tejido conectivo gingival, ligamento periodontal y hueso alveolar, siendo mediada por la interacción de las biopelículas con los mecanismos de defensa del huésped (1-4). Sin duda, esta enfermedad se encuentra indiscutiblemente relacionada con la formación y el desarrollo de las biopelículas en cavidad oral, siendo agravada por la presencia del cálculo dental en relación con la encía $(5,6)$. En este sentido, tanto la perdida ósea, como la pérdida de inserción clínica pueden ser variables en diversos modelos propuestos y puede tardar cierto tiempo en progresar (7-9). Dentro de las opciones para una adecuada valoración periodontal, es necesario tener en cuenta varios aspectos importantes para obtener un correcto diagnóstico: un completo examen visual, el uso de un sonda periodontal adecuadamente calibrada y el examen radiográfico como un complemento y ayuda de los dos primeros $(10,11)$. En relación a este último punto, es necesario establecer que el uso de la técnica del paralelismo ofrece imágenes fiables, lo que permite hacer una evaluación bastante acertada del nivel de altura del hueso alveolar, el estado del ligamento periodontal y la lámina dura (12). Desde el momento en el que se desarrollaron las investigaciones clásicas de Akesson $(13,14)$ y Persson (15) para la estimación de la enfermedad periodontal, se han observado cambios en las tecnologías para la obtención de las imágenes; tanto en radiografías como en tomografías com- putarizadas, este avance ha brindado la oportunidad de crear nuevos esquemas investigativos, donde se verifique el conocimiento científico que ha sido utilizado como verdad durante todos estos años, lo cual necesita ser contrastado o comparado en presencia de la tecnología actual.

Con respecto al hueso alveolar y a la lámina dura, si no se realiza un tratamiento de esta alteración, el progreso natural puede producir con el tiempo disminución de estos soportes del órgano dental, evidenciándose otras características tanto clínicas como radiográficas (16). En este sentido, con la evaluación de la presencia y estado de la lámina dura parece evaluarse el riesgo de la actividad de periodontitis, logrando predecir la evolución de la patología (17-19). El objetivo del presente estudio fue determinar la concordancia y relación entre el nivel de inserción clínica y el examen radiográfico en pacientes con periodontitis crónica que asisten a la facultad de odontología de la Universidad de Cartagena.

\section{MATERIAL Y MÉTODOS}

Se realizó un tipo de estudio descriptivo de corte transversal, a partir de una población diana de 320 pacientes que ingresaron a la institución centro del estudio con diagnóstico de enfermedad periodontal entre 2008 y 2009. La selección de los participantes fue realizada a partir del método aleatorio simple, obteniendo 135 sujetos. Para el cálculo del tamaño de la muestra se utilizó una formula estadística, asumiendo una confianza del 95\%, error relativo del 5\% y frecuencia teórica 
esperada del fenómeno objeto de estudio del $38 \%$ (proporción de sujetos con periodontitis crónica y bolsa periodontal mayor o igual a $4 \mathrm{~mm}$ ).

Previo al proceso de selección, se tuvo en cuenta los datos del diagnóstico periodontal de la historia clínica utilizada en la facultad de Odontología de la institución centro del estudio. En este sentido, todos los sujetos con opción a participar debían tener como diagnóstico confirmado de periodontitis crónica, con bolsas activas periodontales de al menos $4 \mathrm{~mm}$, ser mayor de 16 años de edad. Además, se excluyeron a los sujetos que presentaran cualquier tipo de periodontitis agresiva (20), a los que presentaran antecedentes médicos sistémicos y de terapia periodontal previa, a los que manifestaron ser fumadores, debido a que este hábito podría cambiar la naturaleza del fenómeno en estudio y aquellos que indicaron el uso de antibióticos en los últimos 3 meses antes del estudio. A los 135 participantes se le tomaron un total de 352 radiografías periapicales en 646 dientes y se evaluaron 1292 sitios interproximales.

\section{INSTRUMENTOS}

Se diseñó un formato para recolectar los datos fuente de la medición, el cual fue calibrado a través de una prueba piloto, verificando si estaba acorde con los objetivos del estudio y si los indicadores de cada variable tenían plausibilidad. Además se estandarizaron a tres examinadores a nivel intra e interexaminador, teniendo en cuenta las variables nivel de inserción clínico, pérdida ósea, lámina dura y espacio del ligamento periodontal, comparándose los resultados entre ellos mismos en dos momentos diferentes de tiempo y contra un examinador experto o gold estándar. Para el análisis se tuvo en cuenta un nivel de concordancia kappa $=0,80$ en ambos casos. Al final de este procedimiento fue seleccionado un solo examinador para la medición de la variable clínica y otro examinador diferente para la medición de las variables radiográficas, en ambos casos se realizaron mediciones enmascaradas.

Para la toma de las radiografías periapicales se utilizó la técnica del paralelismo, la cual consiste básicamente en colocar la película paralela al eje longitudinal de los dientes. Se escogió la técnica del paralelismo para este estudio porque minimiza la distorsión dimensional y presenta las estructuras dentarias en su tamaño y posición anatómica (21). El kit para tomar las radiografías en este estudio fue Endoray (22); para estandarizar la técnica se tuvieron en cuenta los siguientes aspectos: la toma de las radiografías fue realizada por un solo investigador del equipo, seleccionado por un experto en radiología, se trabajó con un tiempo de exposición de un segundo, 10 miliamperios y 90 kilovoltios de manera sistematizada en cada una de las tomas de las radiografías. Las radiografías fueron realizadas sin repetición al igual que el revelado y las mediciones, las películas utilizadas fueron marca Kodak ultrarrápidas®.

Una vez obtenidas las radiografías, fueron procesadas de acuerdo a las especificaciones del fabricante. Se procedió a medir la altura del nivel del hueso de la cresta alveolar con ayuda de una regla milimetrada transparente utilizada para la planeación de implantes osteointegrados teniendo en cuenta la distancia entre la línea amelocementaria y el hueso alveolar (23). Para el presente estudio se considera normal una distancia entre la línea amelo cementaría y la cresta ósea de 0 a 3 milímetros. De 4 milímetros en adelante se consideró como perdida ósea (24). Posteriormente se evaluó el tipo de defecto óseo, estado y presencia de la lámina dura y el estado del espacio del ligamento periodontal. Todo esto con la ayuda de un negatoscopio de luz para visualizar claramente las estructuras.

\section{ANÁLISIS ESTADÍSTICO}

Los datos obtenidos fueron agrupados en una base de datos diseñada en Excel versión para Windows 2007, posteriormente se tabularon y se analizaron a través del programa STATA versión para Windows 10.0®. El análisis consistió en el uso de medidas descriptivas a partir de medias, valores máximos y mínimos, al igual que distribuciones de frecuencias absolutas y relativas. Para evaluar la concordancia entre el nivel de inserción clínico y las variables radiográficas, se utilizó la prueba Kappa Cohen y para las relaciones entre estas mismas variables se usaron las razones de odds con intervalos de confianza del $95 \%$, además para establecer significancia se utilizó la prueba $\chi^{2}$ asumiendo un límite de decisión de 0,05.

\section{CONSIDERACIONES ÉTICAS}

Por realizarse mediciones en seres humanos, el presente estudio fue evaluado por el comité de ética de la Universidad de Cartagena, considerándose con riesgo mínimo. Los investigadores utilizaron el consentimiento informado a partir de los lineamientos de la declaración de Helsinki, modificación de Edimburgo 2000 y la resolución 8.430 de 1993 Ministerio de salud. 
República de Colombia. A los participantes se les explicaron las actividades que se les realizarían, para el cumplimiento de los objetivos del estudio, los riesgos a los cuales podrían estar expuestos (toma de radiografías periapicales y medición del surco gingival con sonda periodontal), dejando todo el tiempo en claro que en caso de presentarse, estos serían responsabilidad de los investigadores y cuando el participante no se sintiera cómodo podría retirarse del estudio sin que esto cause diferencia alguna en la atención prestada al fenómeno en estudio.

\section{RESULTADOS}

Los 135 participantes en el presente estudio tenían un promedio de edad de 44,5 años (mínimo= 16; máximo=65), siendo el grupo más frecuente el de 16 a 30 años con el 38\%. En cuanto al sexo, el 52\% eran masculinos y el $48 \%$ femeninos.

La mayor frecuencia de los niveles de inserción fue para los sitios con valores entre 1 y $3 \mathrm{~mm}$ con el 68,0 $\%$. Además se encontró un $27,0 \%$ de pérdida ósea en los sitios examinados. Con respecto al estado de la lámina dura se observó discontinuidad en un 95,0\%. Por otra parte, el espacio del ligamento periodontal se encontró ensanchado en un $96 \%$. En cuanto al tipo de defecto óseo, los verticales se presentaron con un $27,0 \%$ y los horizontales con el $21,0 \%$ (tabla 1 ).

Con respecto al análisis bivariado, para el nivel de inserción y la pérdida ósea se obtuvo un Kappa de 0,58 y una relación estadísticamente significativa, observándose un $77,0 \%$ de niveles de inserción entre 4 y $6 \mathrm{~mm}$ en los sitios con perdida ósea (OR=19, 1; IC 95\%; 13$27 ; p=0,000$ ) (tabla 2). Las demás relaciones puestas a prueba en el presente estudio no fueron estadísticamente significativas y los valores de Kappa estuvieron por debajo de 0,10.

En la tabla 3 se observan los valores correspondientes a la validez de las mediciones radiográficas con respecto al nivel de inserción clínico.

En los niveles de inserción de $1 \mathrm{~mm}$, el 49\% de las observaciones correspondieron a valores de la altura ósea de $2 \mathrm{~mm}$, seguido por un 23,0\% para los valores de $3 \mathrm{~mm}$ y el menor porcentaje correspondió a $4 \mathrm{~mm}$ con el 1,0\% (figura 1). En niveles de inserción de 2 $\mathrm{mm}$ el $36 \%$ correspondió a una altura ósea normal, además, el $28 \%$ de las observaciones presentaron una

\section{TABLA 1.- FRECUENCIA DE LAS VARIABLES DE ESTUDIO (NIVEL DE INSERCIÓN, PÉRDIDA ÓSEA, LÁIMINA DURA, ESPACIO DEL L. PERIODONTAL)}

\begin{tabular}{|c|c|c|c|}
\hline & Número & Frecuencia (\%) & IC $95 \%$ \\
\hline \multicolumn{4}{|l|}{ Nivel de inserción } \\
\hline 4-6 mm ............... & 340 & 32 & $27-37$ \\
\hline $1-3 \mathrm{~mm}$ & 716 & 68 & $64,6-71,4$ \\
\hline \multicolumn{4}{|l|}{ Perdida ósea } \\
\hline ........................ & 283 & 27 & $21,8-32,2$ \\
\hline No & 773 & 73 & $69,9-76,1$ \\
\hline \multicolumn{4}{|l|}{ Lámina dura } \\
\hline Discontinua.... & 1004 & 95 & $93,7-96,3$ \\
\hline 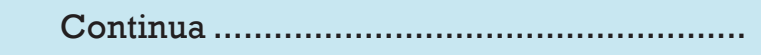 & 52 & 5 & $-0,9-10,9$ \\
\hline \multicolumn{4}{|l|}{ Espacio del 1. periodontal } \\
\hline Ensanchado $\ldots \ldots \ldots \ldots \ldots \ldots \ldots \ldots \ldots \ldots \ldots \ldots \ldots \ldots \ldots \ldots \ldots \ldots \ldots \ldots \ldots \ldots \ldots \ldots \ldots$ & 1014 & 96 & $94,8-97,2$ \\
\hline 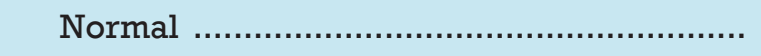 & 42 & 4 & $-1,9-9,9$ \\
\hline \multicolumn{4}{|l|}{ Tipo de defecto óseo } \\
\hline 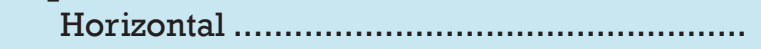 & 216 & 21 & $15,6-26,4$ \\
\hline Vertical & 290 & 27 & $21,9-32,1$ \\
\hline No presente ..................................................... & 550 & 52 & $47,8-56,2$ \\
\hline Total $\ldots \ldots \ldots \ldots \ldots \ldots \ldots \ldots \ldots$ & 1056 & 100 & \\
\hline
\end{tabular}




\begin{tabular}{|c|c|c|c|c|c|}
\hline & \multicolumn{2}{|c|}{ Nivel de inserción clínico } & \multirow{2}{*}{ Kappa } & \multirow{2}{*}{ OR } & \multirow{2}{*}{ IC $95 \%$} \\
\hline & $\begin{array}{c}4-6 \mathrm{~mm} \\
\mathrm{n}=340\end{array}$ & $\begin{array}{c}1-3 \mathrm{~mm} \\
\mathrm{n}=716\end{array}$ & & & \\
\hline \multicolumn{6}{|l|}{ Perdida ósea } \\
\hline ….................... & 122 & 651 & 0,58 & $19,1 *$ & $13,4-27,0$ \\
\hline \multicolumn{6}{|l|}{ Lámina dura } \\
\hline Discontinua & 319 & 685 & & & \\
\hline Continua & 21 & 31 & 0,01 & 1,5 & $0,79-2,66$ \\
\hline \multicolumn{6}{|l|}{ Espacio del 1. periodontal } \\
\hline 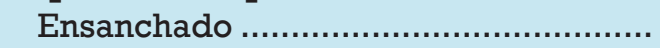 & 330 & 674 & & & \\
\hline 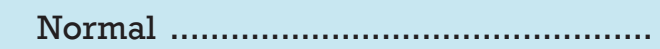 & 10 & 42 & 0,03 & 0,6 & $0,59-1,31$ \\
\hline
\end{tabular}

Valores de significancia: *p $=0,000$

\section{TABLA 3.- VALIDEZ DE LAS MEDICIONES RADIOGRÁFICAS FRENTE AL NIVEL DE INSERCIÓN CLÍNICO}

\begin{tabular}{|c|c|c|c|c|}
\hline & \multicolumn{4}{|c|}{ Nivel de inserción clínico } \\
\hline & Sensibilidad & Especificidad & Predictivo positivo & Predictivo negativo \\
\hline Variables radiográficas & & & & \\
\hline Pérdida ósea ....................... & 64,1 & 90,9 & 77,0 & 84,2 \\
\hline Lámina dura ......................... & 93,8 & 4,3 & 31,8 & 59,6 \\
\hline Espacio del l. periodontal .. & 97,1 & 5,9 & 32,9 & 80,8 \\
\hline
\end{tabular}

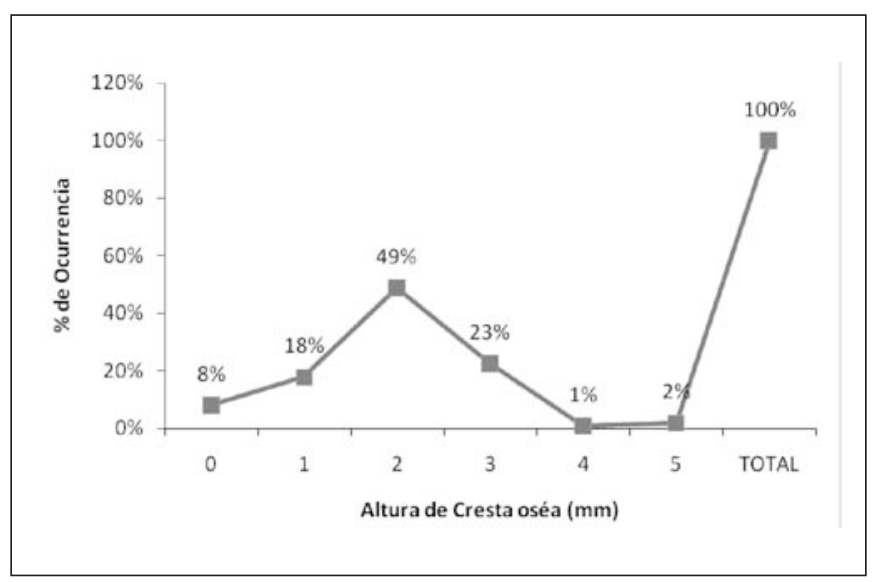

Fig. 1: Frecuencia de la altura de cresta ósea en niveles de inserción de $1 \mathrm{~mm}$. altura ósea de $3 \mathrm{~mm}$, el 5,0\% una altura de $1 \mathrm{~mm}$ y solo el $16,0 \%$ presentaron alturas óseas consideradas como perdida ósea (entre 4 y $7 \mathrm{~mm}$ ) (figura 2). Por otro lado, en niveles de inserción de $3 \mathrm{~mm}$, el $34 \%$ de los casos correspondió a una altura ósea de $2 \mathrm{~mm}$, seguido por un $16 \%$ para una altura de la cresta ósea de $3 \mathrm{~mm}$. Por último, el 21,0\% correspondió a una altura ósea interproximal entre 4 y $5 \mathrm{~mm}$, considerados como pérdidas óseas (figura 3 ).

\section{DISCUSIÓN}

Los resultados del presente estudio, aunque proceden de datos recolectados en un mismo momento de tiempo, lo que no garantiza la temporalidad entre las rela- 


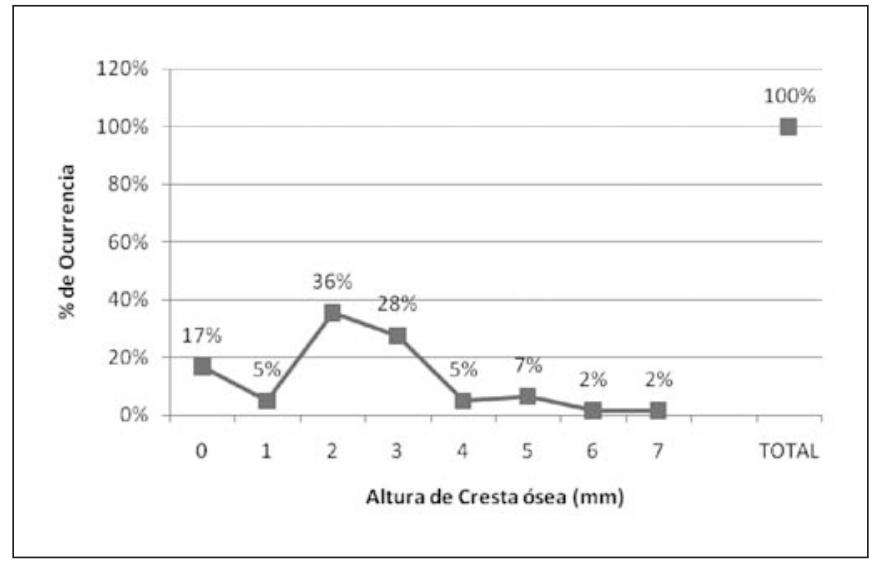

Fig. 2: Frecuencia de la altura ósea en niveles de inserción de $2 \mathrm{~mm}$.

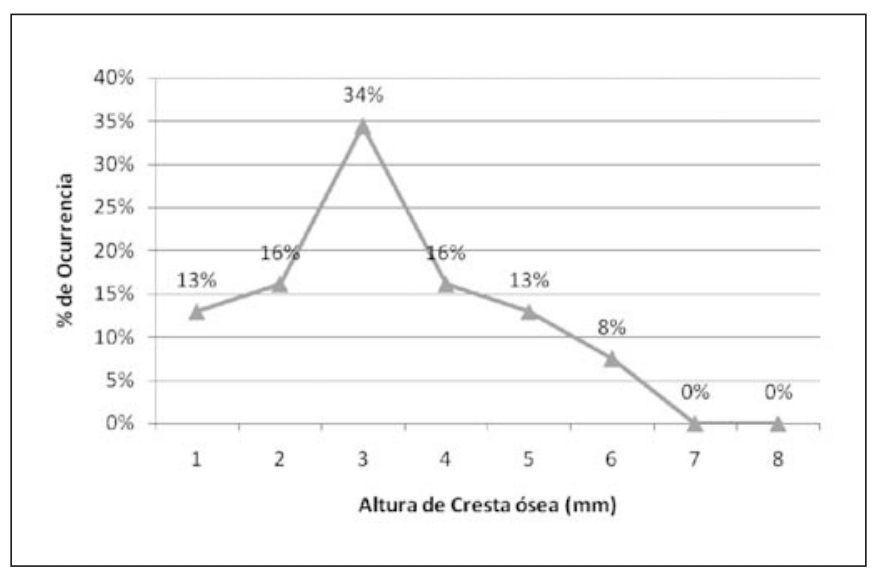

Fig. 3: Frecuencia de la altura ósea en niveles de inserción de $3 \mathrm{~mm}$.

ciones de variables puestas a prueba, si pueden darnos una importante información para la toma de decisiones preventivas y terapéuticas en pacientes con enfermedad periodontal crónica, tomando además del examen clínico periodontal los aspectos radiográficos involucrados.

Según los hallazgos encontrados, en los niveles de inserción entre 1 y $3 \mathrm{~mm}$ se presentaron pocos casos de pérdida ósea radiográficamente, mientras que en niveles de inserción más altos (4 y 5), en la mayoría de los casos se presentó evidencia radiográfica de pérdida ósea. Estos resultados son similares a los encontrados por Díaz (25) en 1998, quienes concluyeron que en bolsas periodontales poco profundas de 3,4 e incluso $5 \mathrm{~mm}$, la imagen radiográfica no evidencia perdida ósea. De la misma manera Park (15) en 2007, reportó que puede existir concordancia en un $82 \%$ entre la imagen radiográfica y los cambios en el nivel de inserción clínica. No obstante en el presente estudio, la máxima concordancia fue del $58 \%$.

El criterio de evaluación utilizado con los participantes en el presente estudio se basó en una distancia de 4 $\mathrm{mm}$ entre la altura de la cresta ósea y la línea amelo cementaría, lo que fue considerada como pérdida ósea, a diferencia de lo establecido por Fukuda en el 2008 (12), con un criterio mucho más estricto de $2 \mathrm{~mm}$, lo que de una u otra forma se refleja en la cantidad de resultados encontrados como positivos para la pérdida ósea, pudiendo de esta forma sobrevalorar el problema, aspecto este que fue controlado en el presente estudio.

La imagen radiográfica fue utilizada como complemento del diagnóstico clínico, debido a que la radiografía dental a pesar de que no revela actividad celular, se pueden evidenciar alteraciones en el tejido calcificado, mostrando los efectos celulares pasados en el hueso y las raíces (26).

Desde esta perspectiva, es claro que el diagnóstico de periodontitis crónica no depende exclusivamente del examen radiográfico, ya que en niveles de inserción entre 1 y $3 \mathrm{~mm}$ no se evidencia pérdida ósea visible. Por otro lado, según los hallazgos encontrados en el presente estudio, la lámina dura no tuvo una relación directa con los niveles de inserción más profundos. Estos hallazgos fueron similares a los encontrados por Greenstein (24) quien reportó que la presencia de la lámina dura no parece tener relación con la presencia de inflamación clínica, sangrado después del sondaje y bolsas periodontales. En este sentido, este concepto es relevante desde la clínica, debido a que la presencia de la lámina dura en las imágenes radiográficas, se utiliza como un indicador de salud periodontal, el cual debe ser corroborado con la utilización sistematizada del examen clínico; uso de sonda periodontal calibrada, para establecer un diagnóstico adecuado desde lo periodontal y clínico $(12,16,23)$.

En un estudio desarrollado por Akesson, Håkansson, Rohlin, y Zöger, (14) los métodos utilizados para la medición periodontal fueron las radiografías panorámicas de diferentes centros odontológicos, además de utilizar radiografías periapicales, contrastando con el presente trabajo, donde se utilizaron exclusivamente radiografías periapicales, tomadas por el mismo técnico en una sola clínica odontológica y evaluadas siempre por uno de los operadores del estudio. A pesar de esto los resultados obtenidos tienen un alto grado de semejanza. En este sentido, no se pretende descono- 
cer la importancia de las antecedentes investigativos previos en este campo disciplinar, pero el papel de la ciencia, obliga a la contrastación permanente de los conocimientos ya establecidos, con el fin de evidenciar cambios desde los diferentes contextos y el uso de las tecnologías modernas y cambiantes.

A partir de este fundamento, la evaluación de la lámina dura y del espacio del ligamento periodontal parece evidenciar el riesgo de actividad de periodontitis en lugares interproximales de los dientes $(6,25)$, lo que permite la corroboración de los sitios con salud periodontal.

Estos resultados permiten realizar un diagnóstico clínico más acertado del estado y avance de la enfermedad periodontal, entendiendo de manera más lógica y eficaz los hallazgos radiográficos; lo cual mejora el manejo y pronóstico de este tipo de patologías.

A la luz de los resultados obtenidos, se concluye que la radiografía dental es un auxiliar del examen clínico periodontal y no un sustituto. Esta combinación puede conducirnos a un diagnóstico más acertado. En este mismo sentido, el diagnóstico de Periodontitis crónica no solo debe depender del examen radiográfico; es evidente que al utilizar esta herramienta de diagnóstico se aumentarían los falsos negativos, presentándose un subregistro de la enfermedad.

\section{AGRADECIMIENTOS}

Al grupo estudiantil "La Célula" de la Facultad de Odontología de la Universidad de Cartagena, por su contribución en la recolección de los datos.

\section{CONFLICTO DE INTERÉS}

Los autores declaramos que no existe conflicto de interés alguno con la institución donde se realizó el estudio. Tampoco se ha recibido dinero o bienes y servicios relacionados con la financiación de este trabajo.

\section{BIBLIOGRAFÍA}

1. Saini R, Marawar PP, Shete S, Saini S. Periodontitis, a true infection. J Glob Infect Dis. 2009 Jul; 1(2): 149-50.

2. D'Aiuto F, Parkar M, Andreou G, Brett PM, Ready D, Tonetti MS. Periodontitis and atherogenesis: causal association or simple coincidence? J Clin Periodontol. 2004 May;31(5):402-11.

3. Schaudinn C, Gorur A, Keller D, Sedghizadeh PP, Costerton JW. Periodontitis: an archetypical biofilm disease. J Am Dent Assoc. 2009 Aug; 140(8):978-86.

4. Reddy MS. The use of periodontal probes and radiographs in clinical trials of diagnostic tests. Ann Periodontol. 1997 Mar;2(1):113-22.

5. Davies RM, Ellwood RP, Volpe AR, Petrone ME. Supragingival calculus and periodontal disease. Periodontol 2000. 1997 Oct;15:74-83.

6. Cobb CM. Microbes, inflammation, scaling and root planing, and the periodontal condition.J Dent Hyg. 2008 Oct;82 Suppl 3:4-9.

7. Wu ZK, Yin YZ. [Evaluation of the remaining periodontal ligament from attachment level]. Shanghai Kou Qiang Yi Xue. Apr;19(2):178-82.

8. Pihlstrom BL. Periodontal risk assessment, diagnosis and treatment planning. Periodontology 2000. 2001;25:37-58.

9. Novaes AB, Jr., Gutierrez FG, Novaes AB. Periodontal disease progression in type II non-insulin-dependent diabetes mellitus patients (NIDDM). Part I-Probing pocket depth and clinical attachment. Braz Dent J. 1996;7(2):65-73.

10. Kaldahl WB, Kalkwarf KL, Patil KD, Molvar MP, Dyer JK. Long-term evaluation of periodontal therapy: II. Incidence of sites breaking down. J Periodontol. 1996 Feb;67(2):103-8.

11. Papapanou PN, Engebretson SP, Lamster IB. Current and future approaches for diagnosis of periodontal diseases. NY State Dent J. 1999 Apr;65(4):32-7.

12. Fukuda CT, Carneiro SR, Alves VT, Pustiglioni FE, De Micheli G. Radiographic alveolar bone loss in patients undergoing periodontal maintenance. Bull Tokyo Dent Coll. 2008 Aug;49(3):99-106.

13. Akesson L, Rohlin M, Hakansson J. Marginal bone in periodontal disease: an evaluation of image quality in panoramic and intra-oral radiography. Dentomaxillofac Radiol. 1989 Aug;18(3):105-12.

14. Akesson $\mathrm{L}$, Hakansson J, Rohlin M, Zoger B. An evaluation of image quality for the assessment of the marginal bone 
level in panoramic radiography. A comparison of radiographs from different dental clinics. Swed Dent J. 1993;17(1-2):9-21.

15. Persson GR. Effects of line-angle versus midproximal periodontal probing measurements on prevalence estimates of periodontal disease.J Periodontal Res. 1991 Nov;26(6):527-9.

16. Rams TE, Listgarten MA, Slots J. Utility of radiographic crestal lamina dura for predicting periodontitis diseaseactivity. J Clin Periodontol. 1994 Oct;21(9):571-6.

17. Van Dyke TE, Tohme ZN. Periodontal diagnosis: evaluation of current concepts and future needs. J Int Acad Periodontol. 2000 Jul;2(3):71-8.

18. Park CH, Abramson ZR, Taba M, Jr., Jin Q, Chang J, Kreider JM, et al. Three-dimensional micro-computed tomographic imaging of alveolar bone in experimental bone loss or repair. J Periodontol. 2007 Feb;78(2):273-81.

19. Pilgram TK, Hildebolt CF, Dotson M, Cohen SC, Hauser JF, Kardaris E, et al. Relationships between clinical attachment level and spine and hip bone mineral density: data from healthy postmenopausal women. J Periodontol. 2002 Mar;73(3):298-301.

20. Armitage GC. Development of a classification system for periodontal diseases and conditions. Ann Periodontol. 1999 Dec;4(1):1-6.

21. Peker I, Alkurt MT. Evaluation of radiographic errors made by undergraduate dental students in periapi- cal radiography. NY State Dent J. 2009 Aug-Sep;75(5): 45-8.

22. Kazzi D, Horner K, Qualtrough AC, Martinez-Beneyto Y, Rushton VE. A comparative study of three periapical radiographic techniques for endodontic working length estimation. Int Endod J. 2007 Jul;40(7):526-31.

23. Perez JR, Smukler H, Nunn ME. Clinical dimensions of the supraosseous gingivae in healthy periodontium. J Periodontol. 2008 Dec;79(12):2267-72.

24. Listgarten MA. Pathogenesis of periodontitis. J Clin Periodontol. 1986 May;13(5):418-30.

25. Díaz A. Periodontal pockets vs bone loss. Revista oficial de la SEPA. 1998; 8;95

26. Khocht A, Zohn H, Deasy M, Chang KM. Screening for periodontal disease: radiographs vs. PSR. J Am Dent Assoc. 1996 Jun;127(6):749-56.

\section{CORRESPONDENCIA}

Antonio Díaz Caballero.

Facultad de Odontología.

Universidad de Cartagena.

Campus de la Salud Zaragocilla.

Cartagena, Bolívar

Colombia

Correo electrónico: adiazcl@unicartagena.edu.co, antoniodiazc@yahoo.com 